\title{
Matriz de cambio y BSC para la gestión de procesos en la fábrica de confecciones Rogger's - Huancayo
}

\section{Change's matrix and BSC for process management on textil factory Rogger's - Huancayo}

\author{
Miguel Fernando Inga Ávila, José Olivera Meza, Freddy Arcos Chuquillanqui (*)
}

\section{RESUMEN}

Debido al desarrollo improvisado y desarticulado de procesos, que genera un flujo de información deficiente, la competitividad organizacional se enfrenta principalmente a barreras internas.

Confecciones Rogger's, empresa huancaína líder en la confección de prendas para caballeros, enfrentaba una situación problema caracterizado por la redundancia e incertidumbre de funciones productivas y logísticas, flujo de información inoportuno e incierto, alto grado de desperdicio de recursos, entre otros, que restringía su competitividad.

Ante esta situación compleja, fue imperativo optimizar los recursos existentes de manera global y sostenida en los sistemas de producción y logística.

El trabajo comprende el diagnóstico de la situación problema y el diseño por procesos del sistema integral de producción y logística mediante la Metodología de Sistemas Blandos, complementado con fundamentos teóricos sobre la Teoría de Restricciones y la Matriz de Cambio en pro de un mayor desarrollo empresarial.

Palabras clave: Cambio Organizacional, MSB, BSC, TOC, Dinámica de Sistemas

\section{ABSTRACT}

Due to the unexpected and disarticulated development of processes, that generates a flow of deficient information, the organizacional competitiveness faces mainly internal barriers.

Textiles Rogger's, wanka company, leader in the article preparation for horsemen, faced a situation problem characterized by the redundancy and uncertainty of productive and logistic functions, flow of inopportune and uncertain information, high degree of waste of resources, among others, that its competitiveness restricted.

Before this complex situation, it was imperative to optimize the existing resources of global and maintained way in the logistic production systems.

The work includes the diagnosis of the situation problem and the design by processes of the integral system of logistic production and by means of the Soft Systems Methodology complemented with theoretical foundations on the Theory of Constrains and the Matrix of Change for a greater enterprise development. Key words: Organisational Change, SSM, BSC, TOC, Dynamic of systems.

\section{PLANTEAMIENTO DEL PROBLEMA}

La actual forma de dirección de las organizaciones, está condicionada a la participación individual, ausencia del enfoque de realimentación, definición deficiente de funciones, monitoreo con escasos indicadores lo cual se manifiesta en la falta de una coordinación orgánica en la ejecución de acciones. La estructura del sistema resultante es ineficaz y deficiente, sin permitir una adecuada toma de decisiones en beneficio del sistema.

Los procesos son llevados a cabo bajo un enfoque lineal, sin considerar con relevancia los efectos sobre los demás procesos y sobre sí mismos, lo cual genera un flujo de información deficiente. 
Las empresas como sistemas sociales se caracterizan por su complejidad generado por el tipo de relaciones que poseen y por la naturaleza ambigua de sus funciones y objetivos. El carácter de las relaciones en los sistemas sociales es bidireccional, debido a que cada miembro del sistema está interconectado con las demás y actúa en función a sus acciones pasadas así como a las acciones pasadas de los demás. Las acciones de las personas están sujetas a las imágenes que estos tienen de su entorno, a las visiones de la organización que poseen y de los objetivos que persiguen; por consiguiente las organizaciones no tienen objetivos claramente definidos. La solución de los problemas en las empresas requiere de una metodología que considere sus características antes mencionada; una metodología basada en el conocimiento previo del sistema y que permita plantear el problema al cual se pretende dar solución. El planteamiento de problemas, como inicio de toda investigación, exige que sea realizado con objetividad y conocimiento de causa, puesto que constituye la guía del trabajo a realizar; por tanto antes de plantear el problema es necesario observar los efectos o síntomas que presenta un sistema social, y así analizar las causas que las generan; sólo de esta manera se evitarán "soluciones sintomáticas» que mejoran la situación del sistema por un pequeño tiempo o peor aún provocan su deterioro.

El proceso de interpretación de las organizaciones consiste en armar alrededor del concepto de totalidad compleja y dinámica un marco teórico referido al fenómeno de las organizaciones humanas. El concepto de totalidad implica considerar a un sistema como tal, con sus interrelaciones, objetivos, estructuras, procesos, políticas, cultura, paradigmas, recursos y capacidades; que conjuntamente generan un comportamiento en constante cambio. Este enfoque conduce a integrar planteamientos teóricos que se adecuen mejor al proceso de interpretación del sistema y solución de sus problemas, llegando a construir un marco teórico apropiado para dicha organización.

\section{METODOLOGÍAS EMPLEADAS}

\section{METODOLOGÍA DE SISTEMAS BLANDOS}

El objetivo general de la metodología de sistemas blandos es el conocimiento gradual de los sistemas sociales y la orientación en el proceso de decisión acerca de las acciones a seguir en beneficio de estos sistemas, proceso cuya eficiencia está sujeta a los criterios empleados.

Las Etapas de la MSB se muestran en la Figura $N^{\circ} 1$ :

Figura $\mathbf{N}^{\circ}$ 1. Metodología de sistemas blandos

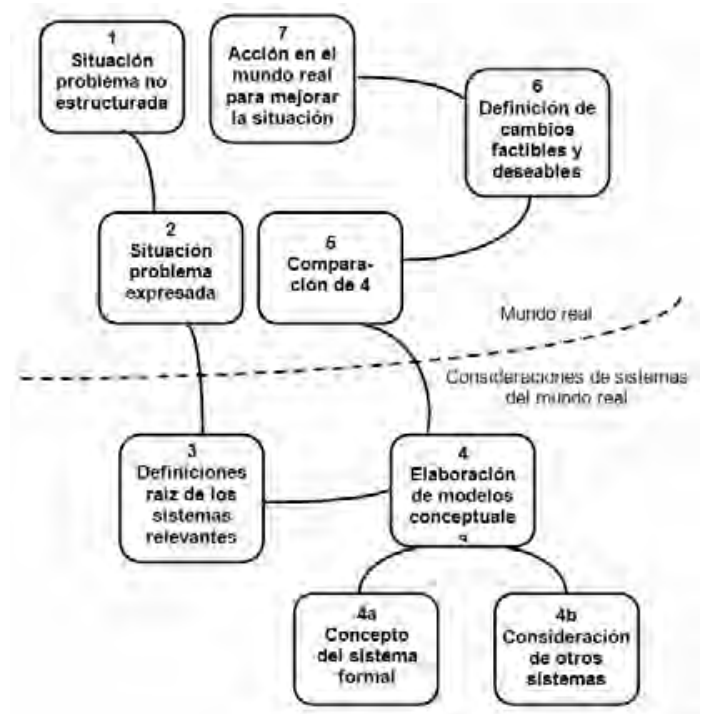

Fuente: Checkland, Peter. System Thinking, Systems Practice. p 188

\section{TEORÍA DE RESTRICCIONES - TOC}

La Teoría de las restricciones fue descrita por Eliyahú M. Goldratt al principio de los 80 en su libro La Meta, y desde entonces ha sido ampliamente utilizada. Es un conjunto de procesos de pensamiento que utiliza la lógica de la causa y efecto para entender lo que sucede y así encontrar maneras de mejorar. Su sustento es que los procesos multitarea, de cualquier ámbito, solo se mueven a la velocidad del ritmo más lento. La manera de acelerar el proceso es utilizar un catalizador y lograr que trabaje hasta el límite de su capacidad para acelerar el proceso en forma integral. La teoría enfatiza la dilucidad, los hallazgos y apoyos del principal factor limitante. 
En la descripción de esta teoría estos factores limitantes se denominan restricciones o «cuellos de botella», que pueden ser un individuo, un equipo, una parte de aparato, una política de la organización, o la ausencia de alguna herramienta o pieza de algún aparato.

Goldratt, resalta la aplicación de la Teoría de las Restricciones (TOC - Theory of Constraints), donde la idea medular es que en toda empresa hay, por lo menos, una restricción. Si así no fuera, generaría ganancias ilimitadas. Siendo las restricciones factores que bloquean a la empresa en la obtención de más ganancias, toda gestión que apunte a ese objetivo debe ser gerenciada focalizada en las restricciones. TOC es una metodología sistémica de gestión y mejora para una empresa. La Meta de cualquier empresa con fines de lucro es ganar dinero de forma sostenida, esto es, satisfaciendo las necesidades de los clientes, empleados y accionistas. Si no gana una cantidad ilimitada es porque algo se lo está impidiendo: sus restricciones.

Contrariamente a lo que parece, en toda empresa existen sólo unas pocas restricciones que le impiden ganar más dinero. Restricción no es sinónimo de recurso escaso. Es imposible tener una cantidad infinita de recursos. Las restricciones, lo que le impide a una organización alcanzar su más alto desempeño en relación a su meta, son en general criterios de decisión erróneos.

a. Definición: La Teoría de Restricciones es una herramienta de gestión que determina donde existen restricciones en la cadena de producción, con el fin de poder optimizar el manejo de los recursos y mejorar la rentabilidad empresarial.

b. Restricción: Es todo aquello que impide alcanzar un objetivo. Son aquellas limitaciones que impiden la utilización de la plena capacidad instalada en la empresa.

\section{Restricciones externas:}

- de mercado o

- de abastecimiento

\section{Restricciones internas:}

- de recursos,

- de comportamiento,

- de procedimientos y de políticas

- logísticas

Las restricciones se comparan a un embudo donde se tiene una entrada en el tubo con gran capacidad, pero a medida que se ingresa en él se va haciendo mas angosto restringiendo la entrada del agua a la parte más estrecha del tubo.

De manera similar, es importante considerar dentro de las organizaciones las limitaciones que se presentan en los procesos observando la secuencia de procesos.

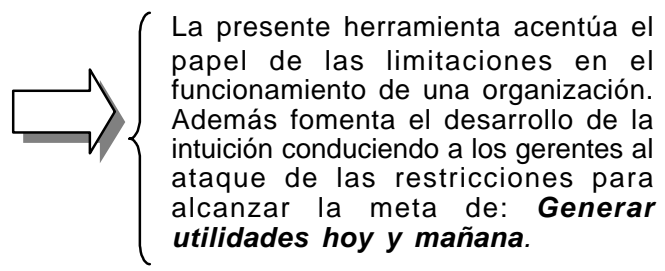

c. Relación entre las medidas financieras y de operación.
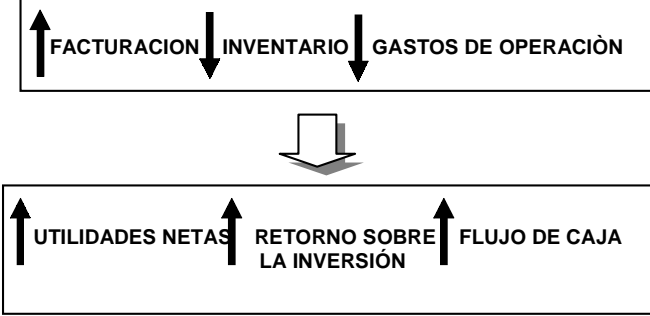

d. Cuellos de botella y recursos no-cuello de botella.

- Un cuello de botella es aquél cuya capacidad es igual o menor que la demanda depositada en él.

- Un no-cuello de botella tiene una capacidad mayor que la demanda puesta en él.

\section{MATRIZ DE CAMBIO 1}

Desde el punto de vista organizacional cualquier proyecto implica cambio, y cualquier cambio

\footnotetext{
1 http://ccs.mit.edu/papers/CCSWP189/CCSWP189.html

Paper: The Matriz of Change. A tool for BPR. Erik Brynjolfsson, Amy Austin Renshaw, Marshall van Alstyne
} 
requiere ser soportado por un proyecto. Para elevar la probabilidad de éxito de una iniciativa de cambio, debemos estar seguros de que el proyecto es entendido y aceptado por toda la comunidad de interesados o stakeholders de la empresa.

Las herramientas tipo Matriz de Cambio (MoC), creadas y promovidas por el MIT, ayudan a caracterizar los rasgos de la gestión del cambio: la viabilidad de los cambios propuestos, la velocidad preferida de ejecución, la mejor secuencia de cambios, la detección de puntos organizacionales críticos en la ejecución de proyectos de inversión.

Lo que se requiere es aprender a ver las métricas de manera más relevantes en periodos apropiados y a emplear procesos formales e informales para medir tales métricas que pueden ser de naturaleza tangible o intangible, a fin de tomar las mejores decisiones.

La Matriz de Cambio o MoC, es una herramienta para administrar el proceso de cambio (derivado de un rediseño). Considera que el éxito depende de la coordinación simultánea entre prácticas de trabajo los cuales permiten alcanzar un cambio coordinado en vez de un cambio aislado.

\section{Etapas de la Matriz de Cambio:}

a. Paso 1 - Identificar los procesos críticos.

b. Paso 2 - Identificar las interacciones del sistema

c. Paso 3 - Identificar las interacciones de la transición

d. Paso 4 - Opinión de los stakeholders

Cada área es importante en la Matriz de Cambio tiene diversas aplicaciones e interpretaciones dentro del proceso de cambio. Vistas conjuntamente brinda pautas útiles acerca de dónde, cuándo, y cuán rápidamente debe implementarse el cambio. El cuadro siguiente muestra en forma genérica estos aspectos. Los gráficos de la matriz tienen diversas y múltiples funciones. El esquema completo de la Matriz de Cambio se observa en la figura siguiente.
Figura $\mathbf{N}^{\circ}$ 2. Esquema de la matriz de cambio

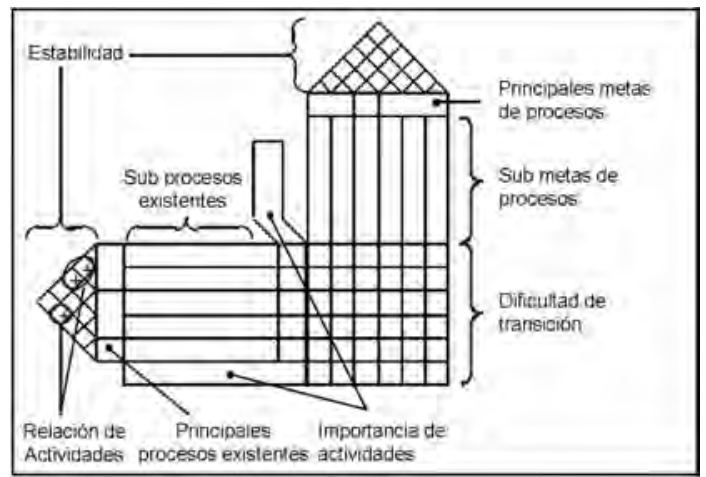

Fuente: Matriz of Change. A Tool for BPR. Brynjolfsson y otros.

\subsection{BALANCED SCORECARD (BSC)}

Lo que uno mide, es lo que logrará. Así, si se mide únicamente el desempeño financiero, solo se obtendrá un buen desempeño financiero. Si por el contrario se amplía la visión, y se incluye medidas desde otras perspectivas, entonces se tendrá la posibilidad de alcanzar objetivos que van más allá de lo financiero.

Esta es la idea fundamental del artículo que en 1992 Robert Kaplan y David Norton escribieran en Harvard Business Review, titulado «The Balanced Scorecard - Measures that Drive Performance» (El Balanced Scorecard Mediciones que llevan a Resultados). Ver Figura $N^{\circ} 3$.

Específicamente, los autores sugieren cuatro perspectivas:

a. Perspectiva financiera: aunque las medidas financieras no deben ser las únicas, tampoco deben despreciarse. La información precisa y actualizada sobre el desempeño financiero siempre será una prioridad. A las medidas tradicionales financieras (como ganancias, crecimiento en las ventas), quizás se deba agregar otras relacionadas como riesgo y costo-beneficio.

b. Perspectiva del cliente: cómo ve el cliente a la organización, y qué debe hacer esta para mantenerlo como cliente? Si el cliente no está satisfecho, aún cuando las finanzas estén marchando bien, es un fuerte indicativo de problemas en el futuro. 
c. Perspectiva interna o de procesos de negocio: cuáles son los procesos internos que la organización debe mejorar para lograr sus objetivos. Debemos preguntarnos: «Para satisfacer a los accionistas y clientes, en qué procesos de negocio debemos sobresalir?»

d. Perspectiva de innovación y mejora: cómo puede la organización seguir mejorando para crear valor en el futuro? Incluye aspectos como entrenamiento de los empleados, cultura organizacional, etc.

Figura $N^{\circ}$ 3. Balanced scorecard y perspectivas

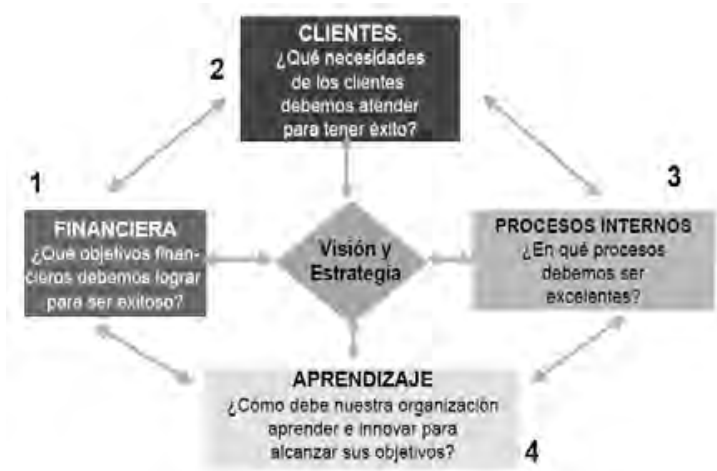

Tomado de: Balanced Scorecard. R. Kaplan y D. Norton. Edit. Gestión 2000, SA

\section{DESARROLLO}

\section{DIAGNÓSTICO DEL SISTEMA}

El problema de la empresa como sistema social es una serie de dificultades interdependientes. El diagnóstico es el soporte para el diseño e implementación de medidas que favorezcan el logro de las metas empresariales.

\section{FUNDAMENTACIÓN PARA LA MATRIZ DE CAMBIO}

\section{a. Situación inicial}

\section{Administración}

- Toma de decisiones centralizadas

- Funciones superpuestas y poco definidas

- Espíritu emprendedor

- Gerencia basada en la experiencia.

\section{Producción}

- Logística poco diferenciada

- Procesos de producción manuales en su totalidad

- Uso deficiente de la capacidad productiva.

- Ineficiente flujo de información entre áreas

- Producción basada en la demanda histórica (30\%)

- Aprendizaje productivo basado en la experiencia

- Ciclo productivo poco standarizado

- Elevado desperdicio de materiales

\section{Marketing y ventas}

- Falta de políticas de atención a los clientes

- Variabilidad en la atención a los clientes

- Falta de espíritu de trabajo en equipo

Aspectos financieros

- Capacidad de inversión limitada

- Capacidad de endeudamiento

\section{b. Situación intermedia}

\section{Administración}

- Toma de decisiones funcionales descentralizadas

- Toma de decisiones estratégicas centralizadas

- Funciones claramente definidas o determinadas

- Inexistencia de MOF

- Espíritu emprendedor directivo

- Organigrama funcional.

- Gerencia basada en la experiencia.

- Comunicación basada en TICs

- Alianzas estratégicas con entidades.

\section{Producción}

- Standarización del ciclo productivo.

- Trabajo cooperativo entre todas las tareas del área

- Labores de logística semi especializadas.

- Procesos de manufactura manuales con asistencia de máquinas especializadas.

- Aprendizaje con experiencia y capacitaciones.

- Desequilibrio en la elaboración de ciertos productos (yampers y casacas) bajo pedido.

- Planeamiento de la producción basada en demanda histórica.

- Disminución de desperdicios.

- Control de calidad en cada proceso. 


\section{Marketing y ventas}

- Control de stock on line por tienda.

- No existe trabajo en equipo.

- Premios y bonos en función de volumen de ventas

- Proyección de ventas sin base técnica

- Desconocimiento de variables de mercado.

- Inexistencia de políticas de atención al público.

- Conocimiento privilegiado del cliente por Venta directa

- Diversificación para venta en bloque.

\section{Aspectos financieros}

- Inversiones en áreas medulares

- Liquidez y fortalecimiento patrimonial

\section{c. Situación deseada}

\section{Administración}

- Empowerment y coaching.

- Funciones claramente definidas o determinadas basadas en el MOF

- Espíritu emprendedor a nivel de toda la empresa

- Estructura organizacional acorde a la naturaleza de la empresa y mercado

- Gerencias profesionalizadas

- Toma de decisiones basadas en Sistemas de Información Gerencial

\section{Producción}

- Procesos productivos y administrativos integrados.

- Labores de logística con sentido estratégico

- Aprendizaje basado en la experiencia y mediante capacitaciones según necesidades.

- Capacidad de respuesta ante demandas fluctuantes o inesperadas.

- Planeamiento de la producción basada en demanda histórica, actual y futura.

- Control de Calidad en todos los procesos.

- Procesos asistidos por herramientas CAD - CAM

\section{Marketing y ventas}

- Control de stock on line por tienda.

- Trabajo sincronizado y en equipo

- Proyección de ventas basado en herramientas técnicas.

- Conocimiento y toma de decisiones basadas en variables de mercado

\section{DISEÑO DEL SISTEMA BASADO EN LA MSB Y TOC}

Un sistema que confecciona y vende prendas de vestir para caballeros, a la luz de los avances técnicos en la industria de la confección y de las características de la demanda, para ser líderes en la Región Central de Perú

\section{Factores relevantes en el diseño del sistema}

a. Los competidores: Precios en el tiempo, Calidad de productos, Capacidad instalada, Posición en el mercado, Formas de distribución.

b. Los proveedores: Productos y servicios que brindan, Calidad, Precios, Facilidades, Formas de abastecimiento, Empatía.

c. La demanda: Gustos y preferencias, Grado de satisfacción, Variaciones en el tiempo, Tendencias

El sistema integral representa la distinción e interconexión de procesos entre el ámbito productivo y logístico de la empresa, estableciendo un sistema para cada ámbito y el flujo de información respectivo; con el objeto que contribuyan a mejorar la productividad y calidad.

\section{ORGANIZACIÓN DEL SISTEMA DE CONFECCIÓN}

Un sistema de la Empresa CR, que de acuerdo a las necesidades del mercado, coordinación con los demás subsistemas y estándares internacionales, confecciona prendas de vestir para caballeros mediante la planificación y realización de procesos basados en criterios de eficiencia y calidad; coadyuvando en el posicionamiento y rentabilidad de la empresa. Los procesos del sistema son:

\section{Planeamiento de la confeción}

La producción de la empresa debe ser planeada en estrecha coordinación con las demás áreas y estudio de la demanda, disponibilidad de recursos y de la economía nacional.

Los beneficios de un adecuado Planeamiento de la Producción incluyen aspectos productivos, administrativos, comerciales y de 
inventarios, tal como se muestra en la Figura $\mathrm{N}^{\circ} 4$.

Figura $N^{\circ}$ 4. Beneficios del planeamiento de la confección

- Se produce lo que se vende más

- Mejor uso del capital de trabajo

- Inventarios con productos de mayor demanda

\begin{tabular}{|c|}
\hline A mejor \\
PLANEAMIENTO \\
DE LA \\
PRODUCCION
\end{tabular}

- Reducción de inventarios de productos con baja demanda

- Adquisición planificada de materiales

- Programación de las operaciones

- Determinación de costos y futuros egresos

- Mejor empleo de la capacidad instalada

Al mismo tiempo, el adecuado Planeamiento de la Producción, requiere la articulación de información proveniente de los diversos procesos de la organización. Ver figura Figura $\mathrm{N}^{\circ} 5$

Figura $N^{\circ} 5$. Coordinación del área de confección con las demás áreas

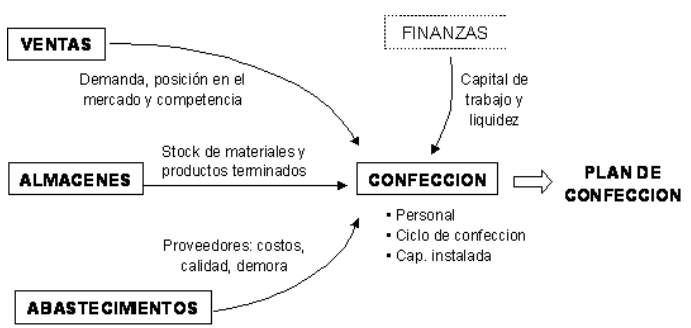

Para que el modelo de integración procedimental mostrado en la figura anterior opere eficientemente, es necesario disponer de algunos elementos. Estos son:

\section{Distribución de operaciones}

La importancia de esta labor, llevó a buscar el equilibrio en la capacidad de cada proceso del ciclo productivo para incrementar el resultado promedio.

\section{Control de calidad}

Un sistema que permita el control de calidad en cada proceso mediante el establecimiento de las especificaciones técnicas de los productos en base a las expectativas de los clientes y la comparación de los resultados de cada proceso de confección con dichas especificaciones. El proceso de Control de Calidad exige la realización de actividades, tal como se muestra en la figura siguiente.

Figura $\mathbf{N}^{\circ}$ 6. Pasos para realizar el control de calidad

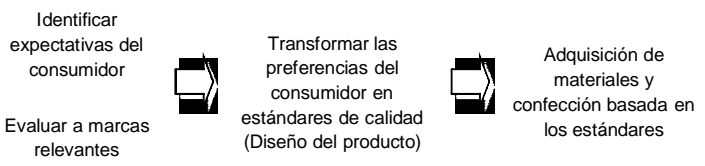

\section{DISEÑO DEL SISTEMA LOGÍSTICO}

Un sistema logístico ágil y adaptable que optimice el flujo de materiales y productos en la empresa.

\section{Abastecimientos \\ El proceso de Abastecimiento involucra la realización y ejecución oportuna de otros procesos relacionados. Esta relación se evidencia en la Figura $\mathrm{N}^{\circ} 7$}

Figura $N^{\circ}$ 7. Ciclo de abastecimiento

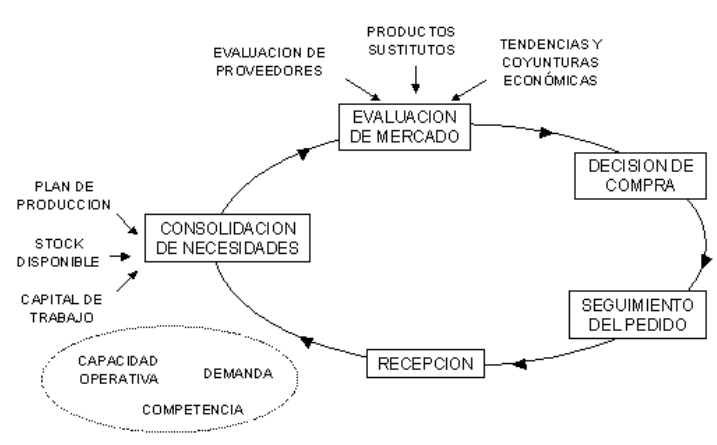

Del mismo modo, el proceso de Abastecimientos está integrado con las demás áreas de la empresa, razón por la cual su adecuado funcionamiento coadyuva a una adecuada respuesta al mercado. Las relaciones exteriores de esta área se muestran en la figura siguiente. 
Figura $N^{\circ} 8$. Coordinación del área de abastecimientos

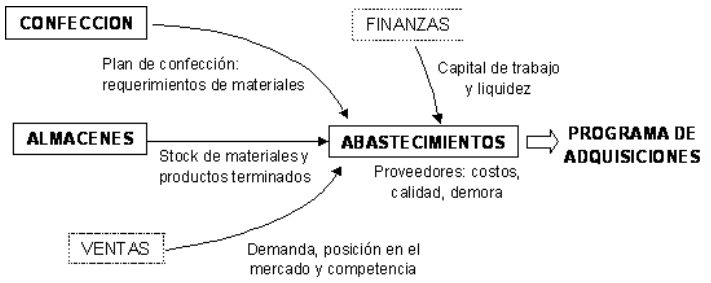

Almacenamiento

a. Objetivo: Internar, acondicionar y proporcionar los artículos a los usuarios en las condiciones requeridas y en el momento preciso, minimizando la inmovilización del capital invertido.

\section{b. Responsabilidades}

- Controlar la exactitud de las existencias de materiales.

- Conservar en buen estado los materiales.

- Reponer oportunamente los materiales básicos.

- Informar oportunamente a confección y abastecimientos sobre los stocks.

\section{c. Actividades:}

- Recepción y almacenamiento de materiales.

- Control de stocks.

- Clasificación de los materiales.

- Ubicación, distribución y cuidado del almacén.

Para la adecuada ejecución del proceso de confección es necesaria la dotación de información oportuna y confiable. Esta necesidad se grafica en la Figura $N^{\circ} 9$.

Figura $\boldsymbol{N}^{\circ}$ 9. Etapas para la confección y flujo de información

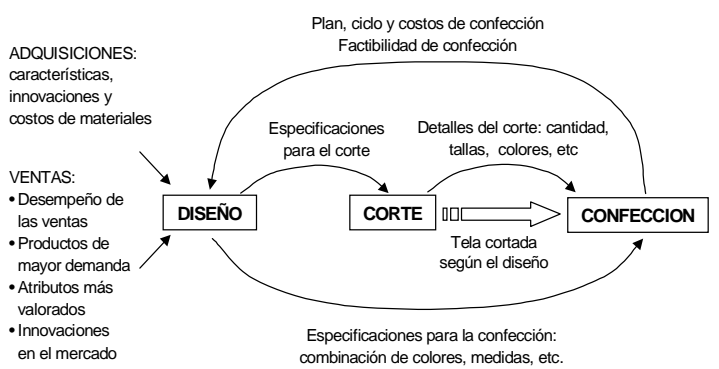

Síntesis del sistema integral y flujo de información

La información y su difusión son fundamentales en la competitividad de la empresa. En el gráfico, se resume cómo debería ser el intercambio de información entre las áreas del sistema integral propuesto. Como se aprecia, hay una mutua dependencia entre todas las áreas de la empresa, y solo trabajando coordinadamente se obtendrá mejores resultados. Ver Figura $N^{\circ} 10$

Figura $\mathbf{N}^{\circ}$ 10. Sistema integral y flujo de información

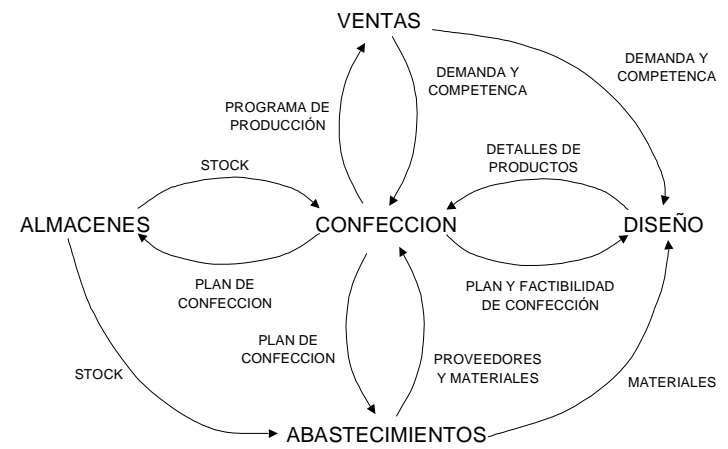

\section{EVALUACIÓN DEL SISTEMA MEDIANTE EL BSC}

La evaluación del comportamiento de las variables relevantes de la Empresa de Confecciones Rogger's se realizó mediante el BSC bajo la Dinámica de Sistemas e implementado en el Software Stella 8.0. Se presenta el tablero mediante el cual se puede ver el comportamiento de los indicadores de cada perspectiva así como señales de advertencia respecto a los objetivos deseados en cada uno de ellos. Ver figura 11.

Figura $\mathbf{N}^{\circ}$ 11. Balanced scorecard de la empresa de confecciones Rogger's

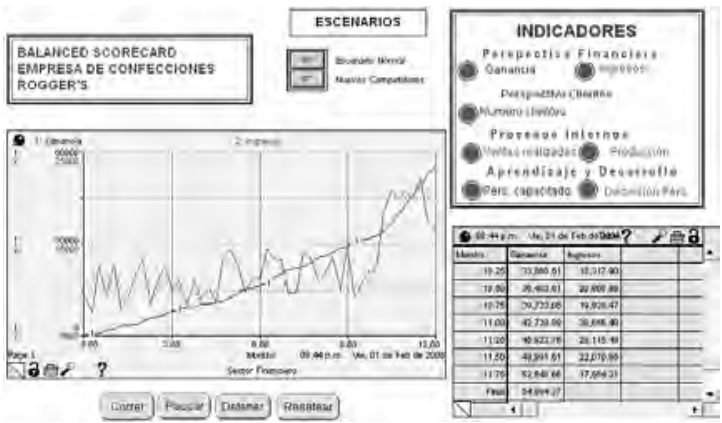


Figura $\mathbf{N}^{\circ}$ 12. Modelo dinámico del BSC de la empresa de confecciones Rogger's

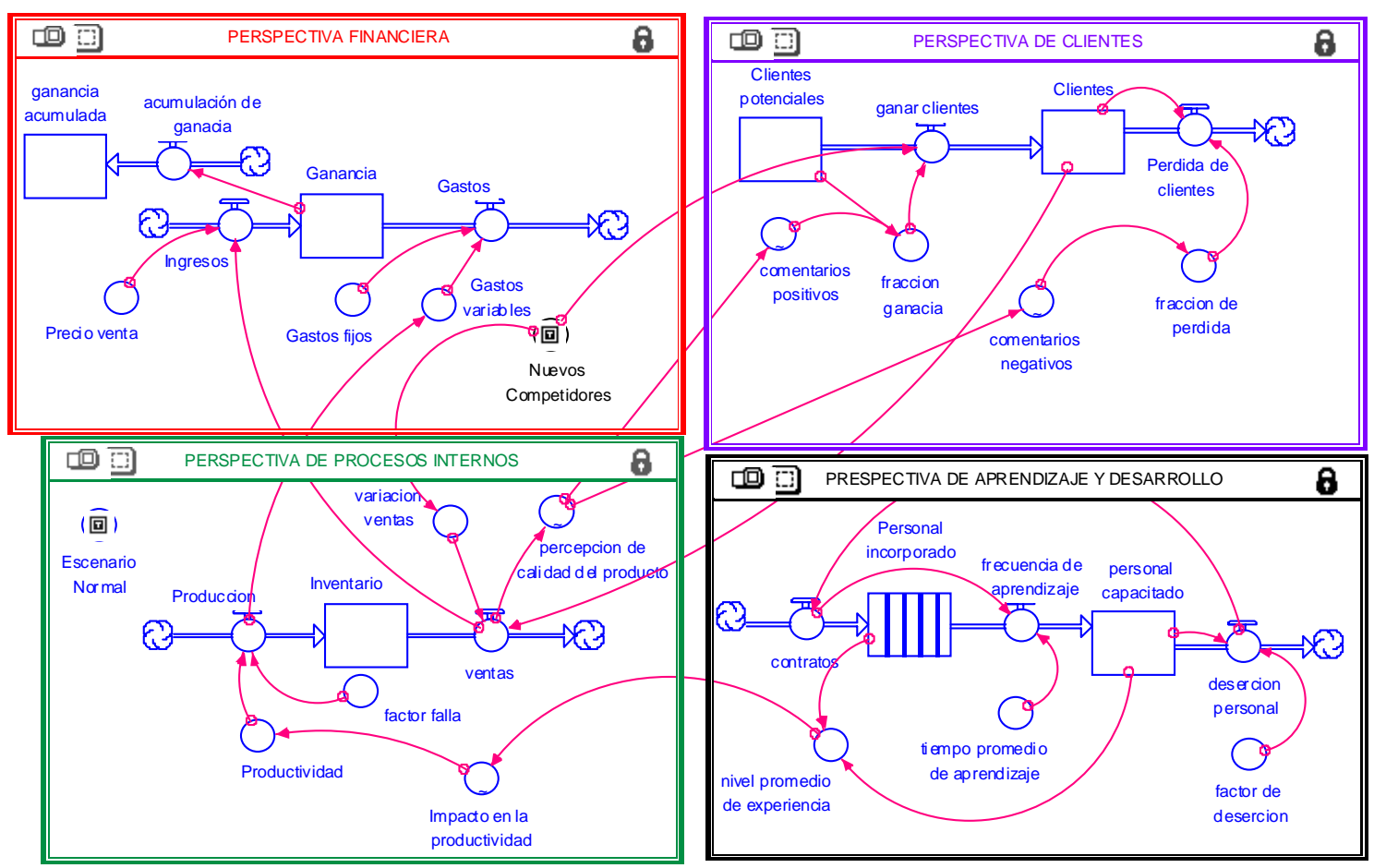

En la Figura 12, se muestra el modelo dinámico del Balanced Scorecard de la empresa a través del Diagrama de Forrester.

\section{RESULTADOS OBTENIDOS DEL SISTEMA PROPUESTO}

a. Los tiempos empleados por cada costurero disminuyen levemente (7\%)

b. El tiempo total en confección disminuye $33 \%$ debido a lo siguiente:

- Cada proceso lo realiza la persona más adecuada.

- Los lotes en proceso se redujeron, circularon 6 lotes uno por cada talla.

- El tiempo de espera por operario se redujo.

c. Incremento inicial de la capacidad de producción diaria de 100 a 130 camisas en promedio sin incremento de personal y equipos.

d. La distribución de operaciones se realizó buscando el equilibrio de las capacidades de cada proceso del ciclo de confección. e. Cada proceso es asignado considerando: rapidez, calidad de trabajo, preferencia y tiempo.

f. Los resultados fueron obtenidos bajo las siguientes condiciones:

- Información anticipada al personal de las operaciones a realizar.

- Preparación anticipada de prefijado y fusionado.

- Disponibilidad de los materiales necesarios

- Estrecha coordinación con los habilitadores.

g. El diseño ha permitido establecer un eficiente flujo de información a nivel de procesos.

h. Monitoreo de las principales variables de la empresa.

\section{CONCLUSIONES}

a. La Matriz de Cambio permitió estructurar y jerarquizar las acciones a llevar a cabo en la empresa. 
b. La gestión de procesos basado en el adecuado flujo de materiales e información bajo la MSB y TOC optimizan los recursos en función de los resultados buscados.

c. El diseño de los procesos es base para la determinación del flujo de información en la organización bajo criterios de objetividad, oportunidad, veracidad y rentabilidad.

d. El BSC permitió monitorear el desempeño de la empresa mediante indicadores relevantes de las cuatro perspectivas.

\section{RECOMENDACIONES}

a. Establecer cargos que impliquen delimitación de funciones y responsabilidades, así como grados de interacción. b. Promover una cultura de innovación.

c. Instruir al personal antes de la realización de una operación nueva.

d. Capacitar al personal en procesos medulares.

e. Estandarizar lugares para mantener el orden.

f. Etiquetar los materiales para facilitar su control.

g. Insertar aplicaciones informáticas para la integración de procesos internos y externos.

\section{AGRADECIMIENTOS}

Al Dr. Walter Barrutia Feijó y al CPC Rogger Inga Díaz.

\section{REFERENCIAS}

Referencias bibliográficas

a. Checkland Peter, Pensamiento de Sistemas. Práctica de Sistemas. México: Grupo Noriega Editores; 1997

b. Goldratt Eliyahú. La Meta. México: Ediciones Castillo: 1998

c. Kaplan R, Norton D. Balanced Scorecard. Edit. Gestión 2000. S.A.

d. Manual de Stella v. 8.0. High Performance Systems. Stella versión 8.0 Technical Documentation. 2003.

Referencias electrónicas

a. Erik Brynjolfsson, Amy Austin Renshaw, Marshall van Alstyne. The Matrix of Change: A Tool for Business Process Reengineering. [monografía en Internet]. Cambridge: MIT Sloan School of Management; 1997. Disponible en: http://ccs.mit.edu/papers/CCSWP189/CCSWP189.html

b. Departamento de Ingeniería Industrial Universidad de Chile. Matriz de Cambio. Disponible: https://www.ucursos.cl/ingenieria/2007/1/IN55A/1/material docente/ objeto/121285

e-mail: miguel_inga@ hotmail.com 